\title{
The development of a brief self-report questionnaire to measure 'recent' Rash Impulsivity: a preliminary investigation of its validity and association with recent alcohol consumption
}

\author{
Dr. Matthew J. Mayhew ${ }^{a}$ and Professor Jane H. Powell ${ }^{b}$ \\ a Department of Psychology, Institute of Psychiatry, King's College London, United Kingdom, \\ SE5 8AF \\ ${ }^{b}$ Department of Psychology, Goldsmiths College, University of London, United Kingdom, \\ SE14 6NW
}

Work carried out at Goldsmiths College, University of London

Correspondence to be sent to: Dr. Matthew J. Mayhew

Postal address: Henry Wellcome Building, Institute of Psychiatry, King's College London, De Crespigny Park, London SE5 8AF

Telephone (office): (+44) 02078480923

Telephone (mobile): (+44) 07983360321

Email address: matthew.1.mayhew@kcl.ac.uk 


\title{
Highlights
}

- We develop a brief self-report scale to assess 'Recent' Rash Impulsivity (the RRIS)

- The RRIS is piloted and subjected to initial validation

- The construct of Recent Rash Impulsivity can be measured via self-report

- Levels of Recent Rash Impulsivity relate to levels of recent alcohol consumption

\begin{abstract}
Background: Traditionally, impulsivity has been regarded as a stable trait. However, a series of longitudinal and behavioural laboratory studies has found that impulsivity can fluctuate within individuals, suggesting that it has a state as well as a trait manifestation. Whilst existing impulsivity questionnaires tap the former, there is no self-report instrument to assess recent fluctuations in impulsivity.
\end{abstract}

Research aims and design: The present study set out to develop and undertake preliminary validation of a measure of 'recent' impulsivity, focusing in particular on Rash Impulsivity. Part of the construct validation of the resulting Recent Rash Impulsivity Scale (RRIS) entailed examining its association with recent alcohol intake, since there are well-documented reciprocal relationships between alcohol consumption and inhibitory control. In developing the RRIS, items from existing trait impulsivity questionnaires were converted into a 'previous two weeks' format. The pilot RRIS was then administered, along with a parallel trait version (Trait Rash Impulsivity Scale; TRIS) and a well-established trait impulsivity measure (the BIS-11; Patton et al., 1995), to two cohorts of first-year undergraduates aged 17 to $25(N=240)$, on two occasions one month apart. Information about habitual and recent alcohol intake was also gathered.

Results: Factor analyses on both the RRIS and TRIS identified two factors: 'Cognitive Impulsivity' (CogImp) and 'Motor Impulsivity' (MotImp). Consistent with the RRIS being sensitive to fluctuations in impulsivity, it was found that, as predicted: i) the RRIS was somewhat less strongly correlated than the TRIS with an established trait measure (the BIS11; Patton et al., 1995); ii) the test-retest stability of 'Total' scores (CogImp and MotImp) was weaker for the RRIS than the TRIS; iii) there was evidence that the RRIS MotImp and Total scales were more strongly predicted by recent alcohol intake than were their trait equivalents; and iv) the RRIS CogImp and Total scales correlated more strongly with their trait equivalents in participants whose alcohol consumption had remained stable recently (relative to their habitual intake), compared to those whose consumption had recently changed.

Conclusions: These data suggest that transient changes in impulsivity can be assessed via self-report, and that the RRIS is sensitive to recent changes in alcohol intake. Subject to more intensive and detailed validation, it is thus promising as a tool for tapping and 
characterising fluctuations in behavioural control and for exploring a range of factors to which this might be associated.

Keywords: State Impulsivity; Recent Impulsivity; Self-Report Questionnaire; Alcohol.

\section{Introduction}

Recent evidence suggests that the tendency to act impulsively is not static but dynamic (Roberts et al., 2001, 2003) and that fluctuations in impulsivity are associated with (changes in) alcohol intake (Littlefield et al., 2009; Field et al., 2010). The primary purposes of the present study were, firstly, to develop a self-report scale to capture recent changes in impulsivity, and secondly, to conduct some preliminary validation of its psychometric properties including its sensitivity to recent alcohol intake.

\subsection{Defining and measuring impulsivity}

Though a multitude of definitions of impulsivity exist, most incorporate the following features: a tendency towards maladaptive behaviour, problems with response inhibition, the gratification of 'automatic' urges and impulses and a relatively low propensity to reflect prior to making decisions - especially those often of a 'risky' nature (Robbins et al., 2012). Thus, it is agreed that impulsivity is not a unitary construct (de Wit \& Richards, 2004; Dalley et al., 2011), the current consensus holding that it consists of at least two broad, distinct yet related, dimensions. Thus, for example, Gullo \& Dawe (2008) have identified the dimensions of 'Reward Drive' (RD) and 'Rash Impulsivity' (RI). These dimensions refer, respectively, to the extent to which one is sensitive to incentives (as manifest in the tendency to engage in appetitive behaviour when exposed to signals of reward) and to the ability to modify or inhibit prepotent (RD-initiated) behaviour in order to avoid potentially aversive consequences.

Existing behavioural and self-report instruments for assessing impulsivity can accordingly be classified as broadly mapping onto these components. Thus, Weafer and de Wit (2013) have subdivided behavioural tasks into those which measure 'Impulsive Action' (similar to RI, and elsewhere described as a deficit of behavioural inhibition) and those which tap 'Impulsive Choice' (similar to RD). The former tasks typically measure the ability to refrain from making a prepotent response when a 'NoGo' or 'Stop' signal is presented (Newman et al., 1985; Logan et al., 1997); those tapping Impulsive Choice quantify either an individual's preference for small, immediate rewards over larger but delayed rewards ('Delay Discounting'; Bickel \& Marsch, 2001; Smith \& Hantula, 2008) or their preference for larger but less certain rewards over smaller but more certain ones ('Probability Discounting'; Green et al., 1999; Poltavski \& Weatherly, 2013). Similarly, with respect to self-report scales, $\mathrm{RD}$ is closely aligned with questionnaires designed to tap the Behavioural Approach/Activation System (BAS; Gray \& McNaughton, 2000); these include Carver and White's (1994) BAS-Drive and BAS-Reward-Responsiveness scales and Torrubia et al.'s 
(2001) Sensitivity to Reward scale. The more numerous instruments tapping the central features of RI include the Barratt Impulsiveness Scale Version 11 (BIS-11; Patton et al., 1995), the Sensation Seeking Scale (SSS; Kolin et al., 1964) and the Impulsiveness scale of Eysenck and Eysenck's (1978) I7.

\section{2. Factors associated with variations in impulsivity}

Impulsivity has traditionally been conceptualised as a stable internal disposition (e.g. McCrae et al., 2000; Costa \& McCrae, 2006). However, in the last decade or so, crosssectional and longitudinal studies have increasingly demonstrated normative (i.e. meanlevel) change across the life-course. Pronounced reductions in impulsivity seem to occur during the transition from adolescence to young adulthood (i.e. between around 18 and 25 years; Arnett, 2000), when conscientiousness, constraint and self-control typically increase (e.g. Roberts et al., 2001, 2003; Donnellan et al., 2007; Blonigen et al., 2008; Vaidya et al., 2008). This progressive reduction in impulsivity continues until around age 50 , at which point it appears to level off somewhat (Roberts \& DelVecchio, 2000; Fraley \& Roberts, 2005).

High levels of trait impulsivity predict problematic alcohol consumption (e.g. see reviews by Crews \& Boettiger, 2009; Aragues et al., 2011). Importantly, a handful of studies suggest there are substantial differences between individuals in the extent to which their impulsivity declines as they mature, and that these differences may be linked with variation in patterns of alcohol consumption. In a longitudinal cohort of students tested from ages 18 through to 35, Littlefield, Sher \& Wood (2009; see also Littlefield et al., 2010) found that: i) there were both normative and individual changes in the level of alcohol involvement across this period; and ii) these changes were associated with changes in a self-reported Rash Impulsivity scale. Interestingly, the sharpest declines in Rash Impulsivity (and alcohol involvement) were observed amongst those aged between 18 and 25 . Subsequent studies by Littlefield, Sher and Steinley (2010) and Quinn, Stappenbeck and Fromme (2011) have reported similar findings.

Research utilising behavioural tasks has demonstrated that the tendency to respond impulsively can fluctuate over shorter intervals, triggered for example by alcohol consumption. Thus, numerous studies have reported acute increases in tests of Impulsive Action following moderate doses of alcohol $(0.4-0.45 \mathrm{~g} / \mathrm{kg})$; these effects on impulsivity are specific, that is, they are not associated with more general impairments of cognitive performance (e.g. Fillmore \& Vogel-Sprott, 1999; de Wit et al., 2000; Marczinski et al., 2005; Rose \& Duka, 2008; Loeber \& Duka, 2009; Miller \& Fillmore, 2013). As noted by Field et al. (2010), however, findings in relation to the effects of alcohol on tests of Impulsive Choice are less consistent. 


\subsection{The present study}

Existing scales are insensitive to possible short-term changes in impulsivity, as they reflect the dominant view of the construct as a stable trait, and accordingly ask about general propensities. For example, the BIS-11 (Patton et al., 1995) presents statements such as, 'I plan tasks carefully' and, 'I am restless at the theatre or lectures', with response options referring to overall frequency ('rarely/never', 'occasionally', 'often' and 'almost always/always'). They thus implicitly encourage the respondent to average over an extended period of time, rather than to focus more narrowly on recent behaviour. Behavioural measures, by contrast, record the individual's 'actual' responses at a precise moment; they are therefore likely to be sensitive to state fluctuations. This difference in the temporal sensitivity of existing behavioural and self-report measures may be one of several factors contributing to the typically weak or non-existent correlations observed between them (e.g. Dom et al., 2006; Cyders \& Coskunpinar, 2011).

There have been no systematic efforts to date to develop a self-report instrument oriented towards recent, rather than long-term, patterns of impulsive behaviour. However, such an instrument would potentially be of utility in illuminating a range of clinical issues, including exploration of possible causal or predictive relationships between impulsivity and addictive behaviour. For example, it might be possible, by monitoring variations in impulsivity, to identify individuals at risk of progressing from social to problematic drinking, or former problem drinkers at risk of relapse, such that interventions can be tailored and effectively timed. An apparent obstacle to developing a 'state' measure comparable to those which exist for anxiety (e.g. the State Anxiety Inventory; Spielberger, 1983) is the intrinsic difficulty of reporting how impulsive one feels at a given moment: impulsive behaviour is by definition unpredictable. However, it is possible to measure recent impulsivity, as distinct from longer-term average tendencies (i.e. trait impulsivity); the present study set out to do just this. In addition to constructing such an instrument, we conducted some preliminary validation. Specifically, we tested whether it had lower testretest stability than corresponding trait measures (as it should be more sensitive to shortterm variations); and, on a similar basis, whether it was more sensitive than trait measures to recent variations in alcohol consumption.

Given that Rash Impulsivity has been consistently found more sensitive than Reward Drive to alcohol consumption (e.g. Littlefield et al., 2009; Miller \& Fillmore, 2013), the new measure was oriented towards items characterising the former facet of impulsivity and is entitled the 'Recent Rash Impulsivity Scale (RRIS)'. Its psychometric properties were compared to those both of an established trait impulsivity measure (the BIS-11; Patton et al., 1995) and a directly corresponding trait version (the Trait Rash Impulsivity Scale; TRIS), in which items corresponding precisely with those in the RRIS were rated by participants against a more extended timescale ('in general', rather than 'within the last two weeks'). Respondents completed questionnaires on two occasions a month apart (Time 1 and Time 2). In the context of findings indicating the most profound changes in impulsivity to occur 
during emerging and young adulthood (e.g. those of Littlefield et al., 2009; 2010; Quinn et al., 2011), the sample was accordingly restricted to individuals between 17 and 25 years of age. It was hypothesised that:

a) The RRIS would correlate more weakly than the TRIS with scores on a 'gold standard' existing trait measure (the BIS-11; Patton et al., 1995);

b) Test-retest correlations, over a 4-week interval, would be lower for the RRIS than the TRIS;

c) RRIS scores would correlate more strongly than TRIS scores with recent changes from typical weekly alcohol consumption;

d) RRIS and TRIS scores would correlate more strongly in participants whose alcohol consumption has remained stable, compared to those whose alcohol consumption has recently changed (compared to typical weekly consumption);

e) Recent changes from typical weekly alcohol consumption would correlate with recent changes in impulsivity, as indexed by subtracting TRIS from current RRIS scores.

\section{Material and methods}

\subsection{Participants}

Two hundred and forty first year Psychology undergraduates aged between 17 and 25 years, all studying at Goldsmiths College, University of London, completed the study and received course credits for doing so. There were no inclusion/exclusion criteria other than being within the above age range, which was chosen to make findings comparable with those from other recent studies investigating impulsivity and alcohol use in young adults.

Participants were drawn from two consecutive undergraduate cohorts, 133 in the first and 107 in the second. There were no differences between the cohorts in terms of age, gender ratio, drinking status, habitual weekly alcohol intake or scores on the three impulsivity scales (i.e. RRIS, TRIS and BIS-11; Patton et al., 1995).

Approval for the study was given by Goldsmiths College Ethics Committee. Participants gave informed written consent after reading an information sheet outlining the study and being assured of confidentiality and that they could terminate their participation at any stage.

\subsection{Measures}

\subsubsection{The Recent Rash Impulsivity Scale (RRIS)}

In developing a measure with the potential to be sensitive to fluctuations in impulsive tendencies over recent and relatively short time periods, it was necessary to identify behaviours or situations that are likely to occur on a day-to-day basis. It was decided to use 'the previous 2 weeks' as the frame of reference because it was felt that this period: i) is short enough for respondents to recall their recent behaviours and experiences 
with reasonable clarity (particularly given that high levels of recent alcohol consumption might impair recollection over more extended periods); ii) is long enough to provide sufficient opportunities for many specific impulsive behaviours to have occurred; and iii) corresponds with the time period employed in other 'state' questionnaires such as the Beck Depression Inventory (Beck et al., 1988). Importantly, respondents may find it harder to base judgements relating to longer periods of time on their recollection of specific incidents or behaviours and thus be more biased by beliefs concerning their general propensity to be impulsive; in that case, there would be a risk of greater overlap with responses on trait scales.

The items of the following widely-used and well-validated trait impulsivity instruments were scanned for items amenable to being converted into a '2-week' response format: the Barratt Impulsiveness Scale (BIS-11; Patton et al., 1995); the EASI-III Impulsivity Scales (Buss \& Plomin, 1984); the Functional and Dysfunctional Impulsivity Scales (Dickman, 1990); the $I_{7}$ Impulsiveness Questionnaire (Eysenck \& Eysenck, 1978); and the Urgency, Premeditation, Perseverance and Sensation Seeking Scales (UPPS; Whiteside \& Lynam, 2001). Some items were unsuitable because they related to attitudes or beliefs rather than specific behaviours (for example, 'Would you agree that almost everything enjoyable is illegal or immoral?'; 'Do you think an evening out is more successful if it is unplanned or arranged at the last moment?'). Others were excluded because of the low a priori likelihood of their having occurred within any given two-week period, for example, 'I change jobs', and, 'I change residences'.

As there was considerable overlap between the 68 items identified from these various measures, redundancy and repetition were minimised by categorising them and then formulating a single question which captured the essence of each category; this yielded 17 questions. Respondents were asked to rate the frequency or probability with which each behaviour had occurred during the previous 2 weeks: 'rarely/never', 'occasionally', 'often' and 'almost always/always'. Some items were reverse scored. Responses were converted to numbers such that for every item 0 represented low impulsivity and 3 high impulsivity.

\subsubsection{The Trait Rash Impulsivity Scale (TRIS)}

This comprised the same items and response options as the RRIS, but was not associated with any specified timeframe. For example, the TRIS item corresponding to the RRIS item, 'In the last two weeks, I have thought carefully before doing and saying things' was simply, 'I think carefully before doing and saying things'.

\subsubsection{The Barratt Impulsiveness Scale (BIS-11; Patton et al., 1995)}

Each item in this 30-item instrument relates to the frequency of some behaviour or attitude and is rated on a 4-point Likert scale, ranging from 'rarely/never' through to 'almost always'. Patton et al. (1995) reported internal consistency coefficients for the total score ranging from 0.79 to 0.83 ; Cronbach's $\alpha$ for the present study was 0.81 . 


\subsubsection{Alcohol Intake}

As participants completed the present questionnaires in the context of an extensive set of measures relating to a number of studies, it was not possible to gather complex data concerning their histories of alcohol use. They were therefore asked just the following two questions, which allowed preliminary testing of hypotheses (c) to (e):

i. How many units of alcohol have you typically consumed in an average week over the last year? They were given information about the number of units in a range of drinks, and their numerical responses were coded into the following categories: $0={ }^{\prime}$ None'; $1={ }^{\prime} 1$ to $4^{\prime} ; 2={ }^{\prime} 5$ to $8^{\prime} ; 3={ }^{\prime} 9$ to $12^{\prime} ; 4=$ ' 13 to $16^{\prime} ; 5={ }^{\prime} 17$ to $20^{\prime} ; 6=$ '21+'.

ii. In the last 2 weeks, how has your alcohol intake compared with your typical weekly intake over the previous year? Responses were: -2 = 'A lot less'; $-1=$ 'A bit less'; $0=$ 'No change'; 1 = 'A bit more'; and 2 = 'A lot more'.

\subsection{Procedure}

Two hundred and forty participants were invited to complete measures in the following order: i) RRIS; ii) demographic information; iii) BIS-11 (Patton et al., 1995); iv) questions about alcohol use; and v) TRIS. The order was fixed in part for pragmatic reasons relating to the fact that these questionnaires were embedded in a highly structured set of other self-report measures relating to a range of studies (though they were grouped together within this larger bundle). Additionally, however, it was considered essential to administer the RRIS before the trait measures so that scores on the former were not biased by a preceding requirement to reflect on general tendencies to act impulsively. A subgroup of 180 participants completed measures (i) and (v) again after a 4-week interval.

\subsection{Data analysis}

\subsubsection{Structure and reliability of the TRIS and RRIS scales}

In order to reduce the data, an exploratory factor analysis (EFA) with varimax rotation was first performed on Time 1 TRIS scores. Confirmatory factor analyses (CFAs) with Maximum Likelihood Ratio estimation were subsequently conducted on TRIS Time 2 and RRIS Time 1 data. The rationale for this approach is that the RRIS, being designed to capture fluctuations in impulsivity, is by definition less likely than trait measures to be stable, both because of state variations and because the 2-week time windows used as the reference periods for RRIS responses are inevitably somewhat prone to variations in the frequencies with which opportunities for the listed behaviours will have arisen. Consequently it is of most theoretical interest to evaluate the internal structure of the trait version, and then test how closely the 'recent impulsivity' version corresponds with it.

Prior to conducting these factor analyses, multivariate outliers were screened using the Mahalonobis distance for all cases (D2). Tabachnick and Fidell (2007) recommend a very 
conservative probability estimate of $\mathrm{D} 2 \leq 0.001$ for a case to be a multivariate outlier; these were accordingly removed prior to analysis.

For the EFA, a preliminary principal components analysis (PCA) was conducted (Cattell, 1966). Bartlett's (1954) test of sphericity and the Kaiser-Meyer-Olkin Measure of Sampling Adequacy (KMO MSA) were used to assess factorisability. The former tests the null hypothesis that the variables are uncorrelated, whilst the KMO MSA compares the magnitudes of the observed correlation and partial correlation coefficients to determine whether the potential factors can be explained by the other variables (Tabachnick \& Fidell, 2007); a KMO MSA of $>0.8$ is considered 'good', and those below 0.7 no better than 'mediocre' (Kaiser, 1974). Following O'Connor (2000), we considered both the Minimum Average Partial (MAP) test (Velicer, 1976) and Parallel Analysis (Horn, 1965) in determining the number of factors to extract. In order to eliminate cross-loadings and aid interpretation of factors, only items which loaded at $\geq 0.50$ were retained (cross-loadings occur when the gap between primary and secondary loadings is smaller than 0.20; Tabachnick \& Fidell, 2007).

For the CFAs, model fit was evaluated using the Root Mean Square Error of Approximation (RMSEA), for which values $\leq 0.08$ indicate 'reasonable' fit (MacCallum et al., 1996); the Standardised Root Mean Square Residual (SRMR), with $\leq 0.05$ indicating 'good' fit (Byrne, 1998; Diamantopoulous \& Siguaw, 2000), and values $\leq 0.08$ being considered 'acceptable' (Hu \& Bentler, 1999); the normed $\chi^{2}$, with values $\leq 2.0$ considered 'very good', and 2.0 to 5.0 'acceptable' (Hair et al., 2008); and the Comparative Fit Index (CFI), for which a value $\geq 0.90$ is 'good' (Hu \& Bentler, 1999).

Internal reliability of the TRIS and RIS was examined using Cronbach's $\alpha$ (Cronbach, 1951). George and Mallery (2003) proposed that $\alpha>0.80$ is 'good', > 0.70 is 'acceptable', > 0.60 is 'questionable' and $>0.50$ is 'poor'. Test-retest reliability and component intercorrelations were assessed via two-tailed Pearson's rs.

\subsubsection{Association-testing}

Directional hypotheses were tested via 2-tailed Pearson correlations. To examine whether two correlations differed in size, Dunn and Clark's (1969) $Z_{1}^{*}$ statistic was used when correlations were dependent and Steiger's (1980) Z statistic when they were independent.

All analyses were performed using the SPSS, with the exception of the CFAs, which were conducted using MPlus Version 4.

\section{Results}

\subsection{Sample characteristics}

Table 1 shows the numbers and proportions of participants with data on each variable at Times 1 and 2 , together with descriptive data pertaining to each. Overall, there were relatively little missing data. 
Table 1: Numbers (and percentages relative to Time 1 total of $N=280$ ) of participants with data on each variable at Time 1 and Time 2 and descriptive data for variable at Times 1 and 2

\begin{tabular}{|c|c|c|c|c|c|c|}
\hline \multirow[b]{2}{*}{ Measure/instrument } & \multicolumn{2}{|c|}{$\begin{array}{c}\text { Time } 1 \\
\text { Total } N=240\end{array}$} & \multicolumn{2}{|c|}{$\begin{array}{c}\text { Time } 2 \\
\text { Total } N=180\end{array}$} & \multicolumn{2}{|c|}{$\begin{array}{c}\text { Time } 1 \text { vs. } \\
\text { Time } 2\end{array}$} \\
\hline & $\begin{array}{l}\text { No. of participants } \\
\text { with complete data }\end{array}$ & $\begin{array}{c}\text { Mean (SD; range) / } \\
\text { Ratio }\end{array}$ & $\begin{array}{l}\text { No. of participants } \\
\text { with complete data }\end{array}$ & $\begin{array}{c}\text { Mean (SD; range) / } \\
\text { Ratio }\end{array}$ & $t / \chi^{2}$ & $p$ \\
\hline Age (years) & $240(100 \%)$ & $19.05(1.49 ; 17-25)$ & $180(75.00 \%)$ & $19.01(1.32 ; 17-25)$ & 0.28 & ns \\
\hline Gender (male/female) & $240(100 \%)$ & $43: 197$ & $180(75.00 \%)$ & $36: 144$ & 0.29 & ns \\
\hline Drinking status (social drinker/abstainer) & $226(94.17 \%)$ & $159: 67$ & $178(74.17 \%)$ & $125: 53$ & 0.001 & ns \\
\hline $\begin{array}{l}\text { Habitual weekly alcohol intake (units per } \\
\text { week during previous } 12 \text { months; 'None' /'1- } \\
4 \text { '/'5-8'/'9-12'/'13-16'/'17-20'/'21+') }\end{array}$ & $226(94.17 \%)$ & $\begin{array}{c}67: 48: 38: 35: \\
18: 9: 11\end{array}$ & $178(74.17 \%)$ & $\begin{array}{c}53: 39: 29: 29: \\
13: 9: 6\end{array}$ & 0.92 & ns \\
\hline $\begin{array}{l}\text { Alcohol intake during previous } 2 \text { weeks } \\
\text { compared to previous } 12 \text { months ('A lot } \\
\text { more' / 'A bit more' / 'No change' / 'A bit } \\
\text { less' / 'A lot less') }\end{array}$ & $220(91.67 \%)$ & $\begin{array}{c}25: 52: 82: 27: \\
34\end{array}$ & $175(72.92 \%)$ & $\begin{array}{c}19: 41: 69: 19: \\
27\end{array}$ & 0.31 & ns \\
\hline RRIS scale $e^{\mathrm{a},+}$ & $240(100 \%)$ & $12.10(3.51 ; 2-23)$ & $180(75.00 \%)$ & $12.19(3.47 ; 2-26)$ & -0.25 & ns \\
\hline TRIS scale ${ }^{\mathrm{a},{ }^{\dagger}}$ & $235(97.92 \%)$ & $11.74(3.83 ; 2-27)$ & $176(73.33 \%)$ & $11.88(3.81 ; 2-27)$ & -0.34 & ns \\
\hline BIS-11 scale & $228(95.00 \%)$ & $\begin{array}{c}65.70(10.11 ; 39- \\
97)\end{array}$ & $180(17.00 \%)$ & $65.46(9.86 ; 39-97)$ & 0.25 & ns \\
\hline
\end{tabular}

\footnotetext{
${ }^{\mathrm{a}}$ Scored from $0-27{ }^{\mathrm{b}}$ Scored from $30-120 ;{ }^{\dagger} 2$-factor scales post-factor analyses.
} 
Time 1 and Time 2 data were drawn from the first and fourth of 4 weekly hour-long data collection sessions in which first-year Psychology undergraduates completed a battery of questionnaires (for a range of studies including the present one) in exchange for course credits. Sixty (25\%) participants from the first session failed to return for the subsequent retest session because, by that point, they had gained the course credits required; this left 180 participants with Time 2 data. There were no differences in age, gender ratio, drinking status, habitual weekly alcohol intake or scores on the three impulsivity scales (i.e. RRIS, TRIS and BIS-11; Patton et al., 1995) between the whole sample of 240 with Time 1 data and this smaller subsample.

\subsection{Structure of the TRIS and RRIS}

\subsubsection{Exploratory factor analysis of Time 1 TRIS data}

Four multivariate outliers were excluded. Within the principal factors extraction, the remaining 231 participants showed no evidence of multicollinearity. The initial PCA confirmed the factorisability of the initial correlation matrix (Bartlett's (1954) Test of Sphericity: $\chi^{2}(136)=893.04 ; p<0.01 ; \mathrm{KMO}$ MSA $\left.=0.82\right)$. Following the procedure described previously, a 2 -factor solution was selected and explained $29.53 \%$ of the variance. As shown in Table 2, five items loaded at 0.50 or higher onto Factor 1 and four on Factor 2; none cross-loaded. Inspection of item content led to their being labelled Cognitive Impulsivity (CogImp) and Motor Impulsivity (MotImp). 
Table 2: Factor loadings, communalities $\left(h^{2}\right)$, eigenvalues and percentages of variance and covariance explained, for exploratory principal factors extraction with varimax rotation on the 17 TRIS items at Time $1(N=231)$

\begin{tabular}{|c|c|c|c|}
\hline Item & $\begin{array}{l}\text { Cognitive } \\
\text { Impulsivity }\end{array}$ & $\begin{array}{l}\text { Motor } \\
\text { Impulsivity }\end{array}$ & $h^{2}$ \\
\hline $\begin{array}{l}\text { I am focused, seeing things through to } \\
\text { the end. }\end{array}$ & 0.74 & 0.15 & 0.57 \\
\hline $\begin{array}{l}\text { I plan work tasks and activities in my free } \\
\text { time carefully. }\end{array}$ & 0.65 & 0.01 & 0.42 \\
\hline $\begin{array}{l}\text { I plan events and activities well ahead of } \\
\text { time. }\end{array}$ & 0.58 & 0.23 & 0.39 \\
\hline $\begin{array}{l}\text { I think carefully before doing and saying } \\
\text { things. }^{\text {a }}\end{array}$ & 0.57 & 0.02 & 0.33 \\
\hline I find it easy to exercise self-control. ${ }^{\text {a }}$ & 0.53 & 0.24 & 0.33 \\
\hline $\begin{array}{l}\text { I encounter problems because I do things } \\
\text { without stopping to think. }\end{array}$ & 0.16 & 0.64 & 0.43 \\
\hline $\begin{array}{l}\text { I become involved with things that I later } \\
\text { wish I could get out of. }\end{array}$ & 0.14 & 0.57 & 0.34 \\
\hline $\begin{array}{l}\text { I tend to jump from one interest to } \\
\text { another. }\end{array}$ & 0.02 & 0.54 & 0.29 \\
\hline I tend to act 'on impulse'. & 0.03 & 0.52 & 0.27 \\
\hline $\begin{array}{l}\text { I am surprised at people's reactions to } \\
\text { things that I do or say. }\end{array}$ & 0.06 & 0.43 & 0.19 \\
\hline $\begin{array}{l}\text { I become so frustrated when waiting, for } \\
\text { example in a shop queue, that I leave. }\end{array}$ & 0.01 & 0.36 & 0.13 \\
\hline I find it easy to concentrate. ${ }^{a}$ & 0.48 & 0.23 & 0.29 \\
\hline $\begin{array}{l}\text { I tend to work quickly, without bothering } \\
\text { to check. }\end{array}$ & -0.37 & 0.27 & 0.20 \\
\hline I become easily bored when working. & 0.23 & 0.43 & 0.24 \\
\hline I find it difficult thinking ahead. & 0.44 & 0.16 & 0.22 \\
\hline I spend more money than I should do. & 0.16 & 0.43 & 0.21 \\
\hline $\begin{array}{l}\text { I get restless when watching things, e.g. } \\
\text { at the cinema / theatre, on television, at } \\
\text { lectures. }\end{array}$ & 0.14 & 0.38 & 0.16 \\
\hline Eigenvalue & 2.62 & 2.40 & \\
\hline Percentage of variance explained & 15.42 & 14.11 & \\
\hline Percentage of covariance explained & 52.19 & 47.81 & \\
\hline
\end{tabular}

All subsequent analyses are based on just these nine items. A Total Score (Total) and separate CogImp and MotImp subscale scores were computed by summing the relevant items.

Internal consistency was conventionally acceptable for TRIS Total $(\alpha=0.73)$ and CogImp ( $\alpha=0.77$ ). Although it was lower for MotImp $(\alpha=0.66)$, this level is considered 
acceptable for research purposes where scales have fewer than 10 items (Holden et al., 1991; Cortina, 1993). This relates to the fact that the internal reliability of a scale increases with the number of items because error variance is increasingly averaged out. Given the small size of MotImp (4 items), we investigated its internal reliability further using the Spearman-Brown Prophecy Formula (Nunnally \& Bernstein, 1994). This takes the observed data and projects what the internal reliability would be in an expanded scale with the same magnitude of inter-correlations. On this basis, if the MotImp subscale comprised four times the present number of items, its internal reliability would be 0.89 . When the same calculation was applied to the Total and the CogImp subscale, the internal reliabilities were 0.92 and 0.93 , respectively. The subscales both correlated highly with the Total $(r=0.84$ and 0.74 for CogImp and MotImp, respectively; $p<0.01$ in each case), but weakly - albeit significantly - with each other $(r=0.26 ; p<0.01)$. Test-retest correlations were moderate for all scores (CogImp: $r=0.54$; MotImp: $r=0.56$; TRIS Total: $r=0.65$; in all cases, $p<0.01$ ).

\subsubsection{Confirmatory factor analysis on the TRIS at Time 2}

Three multivariate outliers were excluded, leaving 173 participants with complete data. Using the criteria described above, the model provided a good fit to the data (RMSEA $=0.08 ;$ SRMR $=0.06 ; \mathrm{CFI}=0.91 ;$ normed $\chi^{2}=2.02$ ). Factor loadings are shown in Table 3.

\subsubsection{Confirmatory factor analysis on the RRIS at Time 1}

Three multivariate outliers were excluded; there were no missing data for the remaining 237 participants. The TRIS model was, overall, a reasonably good fit to the RRIS data: thus, although the CFI of 0.87 fell slightly below $\mathrm{Hu}$ and Bentler's (1999) recommended cut-off of 0.90 , all other fit statistics fell below recommend cut-offs (RMSEA $=0.06$; SRMR $=0.06$; normed $\chi^{2}=1.98$ ). Factor loadings are given in Table 3.

Internal consistency was rather low for the RRIS as a whole $(\alpha=0.64)$ and for both subscales $(\operatorname{Cog} I m p=0.68$; MotImp $=0.53)$, all falling below the conventionally acceptable 0.70. As noted in relation to the TRIS, however, this is not uncommon when scales have fewer than ten items. Application of the Spearman-Brown Prophecy Formula indicated that the observed inter-correlations would yield acceptable internal reliabilities if replicated in versions with four times the present number of items $(0.88$ for the overall scale; 0.89 for CogImp; and 0.82 for MotImp). As for the TRIS, the subscales were both strongly correlated with the Total $(r s \geq 0.73 ; p<0.01)$, and weakly - though significantly - with each other $(r=$ $0.20 ; p<0.01)$. 
Table 3: Items, standardised factor loadings and $Z$ values for confirmatory factor analyses with maximum likelihood ratio estimation on the 9 items loading onto the two subscales of the i) TRIS at Time $2(N=173)$ and ii) the RRIS at Time $1(N=237)$

\begin{tabular}{|c|c|c|}
\hline Item & Loading & $Z$ value $^{\dagger}$ \\
\hline \multicolumn{3}{|l|}{ TRIS Cognitive Impulsivity subscale } \\
\hline I am focused, seeing things through to the end ${ }^{\text {a }}$ & 0.63 & $-^{+\dagger}$ \\
\hline I plan work tasks and activities in my free time carefully ${ }^{a}$ & 0.61 & 4.72 \\
\hline I plan events and activities well ahead of time ${ }^{a}$ & 0.60 & 5.25 \\
\hline I think carefully before doing and saying things ${ }^{a}$ & 0.64 & 4.84 \\
\hline I find it easy to exercise self-control $^{\mathrm{a}}$ & 0.57 & 6.35 \\
\hline \multicolumn{3}{|l|}{ TRIS Motor Impulsivity subscale } \\
\hline I encounter problems because I do things without stopping to think & 0.80 & $-^{++}$ \\
\hline I become involved with things I later wish I could get out of & 0.64 & 6.56 \\
\hline I tend to jump from one interest to another & 0.48 & 5.20 \\
\hline I tend to act 'on impulse' & 0.61 & 6.34 \\
\hline \multicolumn{3}{|l|}{ RRIS Cognitive Impulsivity subscale } \\
\hline I have been focused, seeing things through to the end ${ }^{\text {a }}$ & 0.69 & $--^{++}$ \\
\hline I have planned work tasks and activities in my free time carefully ${ }^{a}$ & 0.54 & 3.45 \\
\hline I have planned events and activities well ahead of time ${ }^{a}$ & 0.51 & 4.36 \\
\hline I have thought carefully before doing and saying things ${ }^{a}$ & 0.50 & 3.42 \\
\hline I have found it easy to exercise self-control ${ }^{\mathrm{a}}$ & 0.52 & 6.70 \\
\hline \multicolumn{3}{|l|}{ RRIS Motor Impulsivity subscale } \\
\hline I have encountered problems because I did things without stopping to think & 0.76 & $-{ }^{++}$ \\
\hline I have become involved with things that I later wished I could have got out of & 0.36 & 3.38 \\
\hline I have tended to jump from one interest to another & 0.36 & 2.51 \\
\hline I have tended to act 'on impulse' & 0.43 & 2.79 \\
\hline
\end{tabular}

${ }^{a}$ Indicates item is reverse-scored; ${ }^{\dagger}$ Values above 1.96 are considered statistically significant; ${ }^{\text {t+ }}$ Not estimated as loading set to fixed value (i.e. 1.00). 


\subsection{Hypothesis-testing}

Each of the hypotheses is recapitulated or paraphrased below, followed by the corresponding analysis/analyses.

3.3.1. Hypothesis a): The RRIS will correlate more weakly than the TRIS with scores on a 'gold standard' existing trait measure (the BIS-11; Patton et al., 1995)

In the 223 participants with complete data, the correlation between the BIS-11 and the TRIS-Total $(r=0.75 ; p<0.01)$ was significantly greater than that between the BIS-11 total and the RRIS-Total $(r=0.67 ; p<0.01)\left(z_{1}{ }^{*}=2.35 ; p=0.02\right)$. Although the difference in the size of the correlations was not large, this was consistent with the hypothesis.

3.3.2. Hypothesis b): Test-retest correlations, over a 4-week interval, will be lower for the RRIS than the TRIS

One hundred and seventy three participants had complete data for these analyses. Consistent with the hypothesis, the test-retest correlation for the Total score was significantly lower for the RRIS than for the TRIS ( $r S=0.53$ vs. 0.65 , respectively; $Z=2.19 ; p=$ 0.03). There was a similar trend for the MotImp subscale (RRIS: $r=0.46$; TRIS: $r=0.56$ ), though this difference fell short of significance $(Z=1.53 ; p=0.13)$. However, there was no hint of any difference for the CogImp subscale (RRIS: $r=0.51$; TRIS: $r=0.52$ ).

3.3.3. Hypothesis c): RRIS scores will correlate more strongly than TRIS scores with recent changes from typical weekly alcohol consumption

In testing hypotheses c) to e), analyses excluded 67 participants who reported zero alcohol consumption over the previous 12 months. One hundred and fifty five of the remaining participants provided complete data.

The hypothesis was supported for MotImp, for which the RRIS score correlated more strongly than the TRIS score with recent changes in alcohol intake ( $r s=0.37$ vs. 0.19, respectively; $Z_{1}{ }^{*}=2.56 ; p=0.01$ ). The same was true for the Total scale (RRIS: $r=0.28$; TRIS: $\left.r=0.14 ; Z_{1}^{*}=2.19 ; p=0.03\right)$. This was not the case for CogImp, however, where there was no significant correlation with recent changes in alcohol consumption for either RRIS or TRIS scores (both $r s<0.10 ; \mathrm{ns}$ ).

3.3.4. Hypothesis d): RRIS and TRIS scores will correlate more strongly in participants whose alcohol consumption has remained stable, compared to those whose alcohol consumption has recently changed (compared to typical weekly consumption)

Thirty-three participants reported no recent change in their alcohol intake (compared to their intake during the previous 12 months; 'Non-Changers'), whilst 122 reported either increases or decreases ('Changers'). The hypothesis was supported for CogImp, with the correlation between TRIS and RRIS scores being stronger for Non-Changers than Changers ( $r s=0.82$ vs. 0.56 , respectively; $Z=2.56 ; p=0.01$ ). The hypothesis was also 
supported for the Total scale (Non-Changers: $r=0.83$; Changers: $r=0.63 ; Z=2.19 ; p=0.03$ ). This was not the case, however, for MotImp (Non-Changers: $r=0.56$; Changers: $r=0.57 ; Z=$ -0.07 ; ns).

3.3.5. Hypothesis e): Recent changes from typical weekly alcohol consumption will correlate with recent changes in impulsivity, as indexed by subtracting TRIS from current RRIS scores

The 'alcohol change score' (ACS; -2 to +2) was correlated with the derived 'impulsivity change' indices, computed for Total, CogImp and MotImp scores. In the 155 participants with complete data, the correlation between MotImp 'change' and ACS was small but significant $(r=0.17 ; p=0.04)$; thus, consistent with the hypothesis, recent changes in motor impulsiveness were weakly linked with corresponding recent changes in alcohol consumption. The correlation between the Total 'change' and ACS demonstrated a similar relationship, but just fell short of significance $(r=0.15 ; p=0.06)$. This was not the case for CogImp, however ( $r=0.05$; ns).

\section{Discussion}

Premised on a literature suggesting that impulsivity may fluctuate over time, and that such changes are associated with alcohol consumption (e.g. Roberts et al., 2001, 2003; Littlefield et al., 2009; Field et al., 2010), we set out to develop and pilot a new instrument (the RRIS) for assessing individuals' impulsivity over the immediately preceding two weeks. The key findings of the present study were that: i) the RRIS correlated less strongly than its trait equivalent (the TRIS) with an existing trait measure (the BIS-11; Patton et al., 1995); ii) test-retest stability was weaker for the RRIS than the TRIS Total scale; iii) the RRIS Total and Motor Impulsivity scales were more strongly predicted by recent alcohol intake than were their trait equivalents; and iv) the RRIS Cognitive Impulsivity and Total scales correlated more strongly with their trait correspondents in those whose alcohol consumption had remained stable recently (relative to their habitual intake), compared to those whose consumption had recently changed.

\subsection{Structure and validity of the TRIS and RRIS scales}

Factor analyses of the newly-developed 'recent' and 'trait' Rash Impulsivity scales (respectively, the RRIS and TRIS) revealed two distinct factors, the first defined by five items and the second by four; eight items with loadings below 0.5 on either factor were eliminated in the interests of creating a short and easy-to-administer instrument. Cognitive Impulsivity (CogImp) manifested as planfulness and greater control, as illustrated by its two highest-loading items: 'I plan work tasks and activities in my free time carefully' and, 'I am focused, seeing things through to the end'. Motor Impulsivity (MotImp) reflected the tendency to behave rashly, without considering potential negative consequences; for example, 'I encounter problems because I do things without stopping to think' and, 'I become involved with things that I later wish I could get out of'. Reflecting the way in which 
items were derived, the content of the subscales resembles that of the subscales in some of the source instruments. In particular, CogImp is similar to BIS-11 Non-Planning Impulsiveness (Patton et al., 1995), UPPS Premeditation (Whiteside \& Lynam, 2001) and EASI-III Decision Time (Buss \& Plomin, 1984); whilst MotImp resembles BIS-11 Motor Impulsiveness (Patton et al., 1995), UPPS Urgency (Whiteside \& Lynam, 2001) and EASI-III Inhibitory Control (Buss \& Plomin, 1984).

For questionnaire development, the present sample size $(N=231)$ is considered 'fair' (Comrey \& Lee, 1992); and both the exploratory and confirmatory factor analyses (CFAs) described here, based on participant to variable ratios of around 200:9, are likely to have been robust.

The total variance explained by these two factors was relatively low at $29.53 \%$. This may reflect the putative multidimensionality of impulsivity (de Wit \& Richards, 2004; Dalley et al., 2011) and constraints on the selection of items for the pilot instrument. Thus, only certain types of items lent themselves to the response format (frequency of occurrence within the last two weeks). Although we focused specifically on so-called 'Rash Impulsivity' (Gullo \& Dawe, 2008), which was of particular interest given previous evidence that it is more affected than Reward Drive by alcohol consumption (e.g. Fillmore \& Vogel-Sprott, 1999; Littlefield et al., 2009; 2010), it is likely that responses to individual items were also influenced by (or indicative of) other factors including disparate aspects of impulsivity. It would be interesting for future development of this or similar scales to explore whether the inclusion of items more systematically tapping other elements of impulsivity (e.g. Reward Drive) would yield a different factor structure and account for a higher proportion of the variance.

Internal consistency was conventionally acceptable (> 0.70; George \& Mallery, 2003) for the TRIS Total scale and the TRIS CogImp subscale. Although as were below 0.70 for the other scales, their values were nevertheless deemed acceptable for research purposes as these scales all consisted of fewer than 10 items (Holden et al., 1991; Cortina, 1993); and the same magnitude of inter-item correlations would have yielded reliability coefficients markedly exceeding the conventional 0.70 level if replicated with more items. There was a tendency for the RRIS to show slightly weaker internal consistency generally than the TRIS, possibly reflecting the narrow time-window within which the frequency of behaviours was rated. Thus, it is less likely that all of the situations itemised in the RRIS will have occurred within the specific fortnight tapped by the questionnaire than that they will all have occurred in the (much) more extended period tapped by the TRIS. If some situations have simply not been experienced by some participants, this will of course mean that associations between response tendencies in those situations cannot be detected as robustly as when respondents are asked to reflect on more protracted histories. Interestingly, however, for no RRIS items did more than $23 \%$ of respondents give a 'never' response; this suggests that every scenario had been experienced by the majority of respondents at least once. Given that this 'baseline occurrence' issue may nevertheless be an intrinsic difficulty for 'recent' or 'state' instruments which refer to 'real-life' experiences within a narrow time-window, it is 
cautiously encouraging that $\alpha$ s for both the overall TRIS and the CogImp subscale were nevertheless above 0.60 , and that the factor structure of the RRIS corresponded well with that of the intrinsically more stable TRIS.

It was hypothesised that, since the RRIS was expressly designed to be sensitive to recent fluctuations in state, it should correlate less strongly than its trait equivalent (the TRIS) with the BIS-11 (Patton et al., 1995), a well-established trait impulsiveness measure. This was borne out. On the same basis, it was hypothesised that the RRIS should show weaker test-retest stability than the TRIS; a significant difference was confirmed for the Total score, and there was a trend for the MotImp subscale, though not for the CogImp subscale. Whilst encouraging, it is possible that these findings could to some extent reflect the weaker internal consistency of the RRIS than the TRIS, and further exploration of the psychometric properties of these instruments are needed. Future studies might, for example, explore relationships with a wider range of existing impulsivity measures, and within a more demographically varied sample than that tested here (all Psychology students aged 25 or under and predominantly female).

\subsection{Relationships of the TRIS and RRIS to indices of alcohol consumption}

Additional evidence of the utility of the RRIS derives from findings suggesting that, as theoretically predicted, it is more sensitive than its trait counterpart to variations in recent (compared to habitual) alcohol consumption. This was particularly the case for Motlmp, with self-reported recent changes in level of drinking being reflected in corresponding recent changes in self-reported tendencies to be more generally impulsive or disinhibited. The same patterns were observed for the Total scale. This mirrors experimental findings (e.g. Fillmore \& Vogel-Sprott, 1999). The same was not true for CogImp, however. It may be that alcohol has more pronounced or striking effects on motor behaviour than cognitive functioning, or that the former effects are simply more memorable. This is plausible, given that there were few very heavy drinkers in this sample and that social drinkers typically consume alcohol in relaxed evening periods, rather than during daytime working hours, when cognitive planfulness/control functions are most engaged. Interestingly, however, relative to a subgroup of participants whose drinking patterns were stable, 122 participants whose drinking had recently increased or decreased showed a stronger tendency to report parallel changes in Cognitive Impulsivity (but not Motor Impulsivity) over the same period. (The same was also true for the Total scale.) This pattern suggests, contrary to the previous observation, that recent drinking did in fact destabilise cognitive impulsivity.

Whilst the possibility that some of these findings are spurious cannot be excluded, the present findings do give tentative support to the proposition that the RRIS is more sensitive than the TRIS to the effects of recent alcohol consumption. This merits more detailed exploration in a larger and more diverse sample, employing more systematic measures of alcohol intake than was feasible in this pilot study. If some or all of the correlations observed here are replicated, it is worth noting that the causal pathways linking alcohol consumption with impulsivity are likely to be bi-directional (Quinn et al., 2011). 
Thus, whilst alcohol clearly has acutely disinhibiting effects on behaviour, longitudinal evidence indicates that trait impulsivity is itself a risk (or at least, predictive) factor for future alcohol consumption (e.g. Crews \& Boettiger, 2009; Aragues et al., 2011). In relation to the correlations observed here, it could equally be that variations in impulsivity influenced drinking behaviour or that changes in their drinking increased, attenuated or simply mirrored their propensity to act impulsively in more general ways.

There are other questions it would be desirable to investigate in future validation of the RRIS. For instance, the ability to complete it accurately may be reduced if relatively heavy drinking during the preceding two weeks has impaired a respondent's recollection of at least part(s) of that period. Thus, for example, binge drinkers may simply not remember specific incidents of impulsive or disinhibited behaviour which occurred when they were intoxicated. This would have the consequence of attenuating the correlations between RRIS scores and recent alcohol intake such that they under-represent reality. The effects of alcohol, or indeed other factors, impairing memory are an intrinsic challenge to self-report measures which require recollection of recent events, but are likely to be particularly acute when the behaviours of interest are - as with impulsivity - likely to be associated with factors which compromise reliable memory for them. It would thus be of interest to investigate the associations between self-reported and observer-reported impulsive behaviours; this could certainly be explored in experimentally controlled situations. In practice, the sampling method used in the present study resulted in the inclusion of very few heavy drinkers, so although the potential memory bias is unlikely to have exerted much effect on findings, it is also the case that there was a fairly restricted range of drinking and, possibly, impulsivity, within which to examine associations. It would clearly be desirable to carry out further studies within more heterogeneous samples. In relation to this, it is relevant to note that comparison of different age groups would be of considerable interest. Thus, longitudinal studies have found trait impulsivity to undergo profound alteration during late adolescence to early adulthood (e.g. Arnett, 2000; Roberts et al., 2001, 2003), reducing and reaching a stable plateau by around the age of 50 (Roberts \& DelVecchio, 2000; Fraley \& Roberts, 2005).

Finally, it was suggested in the Introduction that the RRIS might be more closely related than trait impulsivity measures to indices from behavioural tasks, which tap impulsive response tendencies at a particular moment in time. This would be straightforward to test in a design administering a combination of self-report and behavioural tests in parallel. If RRIS scores were to indeed show a closer correspondence than the TRIS or other trait instruments with behavioural indices, it would not only help to explain the weakness of observed inter-correlations between trait and behavioural indices of impulsiveness, but further validate the RRIS as a new tool of potential utility in theoretical and applied contexts. 


\section{Role of funding sources}

Mayhew conducted this research as part of his doctoral work under Powell's supervision, funded by a joint Economic and Social Research Council and Medical Research Council $1+3$ interdisciplinary studentship (award reference number: ES/1902511/1).

\section{Contributors and acknowledgments}

Both authors contributed to all aspects of the design, statistical analysis and preparation of the manuscript. Both authors have approved the final manuscript. We are grateful to Alan Pickering and Andrew Cooper for statistical advice.

\section{Conflict of interest}

No conflict declared.

\section{References}

Aragues, M., Jurado, R., Quinto, R. \& Rubio, G. (2011). Laboratory paradigms of impulsivity and alcohol dependence: A review. European Addiction Research, 17, 64-71.

Arnett, J. J. (2000). Emerging adulthood - a theory of development from the late teens through the twenties. American Psychologist, 55, 469-480.

Bartlett, M. S. (1954). A note on the multiplying factors for various chi-2 approximations. Journal of the Royal Statistical Society Series B-Statistical Methodology, 16, 296-298.

Beck, A. T., Steer, R. A. \& Garbin, M. G. (1988). Psychometric properties of the beck depression inventory - 25 years of evaluation. Clinical Psychology Review, 8, 77-100.

Bickel, W. K. \& Marsch, L. A. (2001). Toward a behavioral economic understanding of drug dependence: Delay discounting processes. Addiction, 96, 73-86.

Blonigen, D. M., Carlson, M. D., Hicks, B. M., Krueger, R. F. \& lacono, W. G. (2008). Stability and change in personality traits from late adolescence to early adulthood: A longitudinal twin study. Journal of Personality, 76, 229-266.

Buss, A. H. \& Plomin, R. (1984). Temperament: Early developing personality traits. Hillsdale, New Jersey: Lawrence Erlbaum Associates.

Byrne, B. M. (1998). Structural equation modeling with lisrel, prelis and simplis: Basic concepts, applications and programming. Hillsdale, New Jersey: Erlbaum.

Carver, C. S. \& White, T. L. (1994). Behavioral-inhibition, behavioral activation, and affective responses to impending reward and punishment - the bis bas scales. Journal of Personality and Social Psychology, 67, 319-333. 
Cattell, R. B. (1966). The scree test for the number of factors. Multivariate Behavioral Research, 1, 245-276.

Comrey, A. L. \& Lee, H. B. (1992). A first course in factor analysis (2nd ed.). Hillsdale, New Jersey: Lawrence Erlbaum Associates.

Cortina, J. M. (1993). What is coefficient alpha - an examination of theory and applications. Journal of Applied Psychology, 78, 98-104.

Costa, P. T. \& McCrae, R. R. (2006). Age changes in personality and their origins: Comment on roberts, walton, and viechtbauer (2006). Psychological Bulletin, 132, 26-28.

Crews, F. T. \& Boettiger, C. A. (2009). Impulsivity, frontal lobes and risk for addiction. Pharmacology Biochemistry and Behavior, 93, 237-247.

Cronbach, L. J. (1951). Coefficient alpha and the internal structure of tests. Psychometrika, $16,297-334$.

Cyders, M. A. \& Coskunpinar, A. (2011). Measurement of constructs using self-report and behavioral lab tasks: Is there overlap in nomothetic span and construct representation for impulsivity? Clinical Psychology Review, 31, 965-982.

Dalley, J. W., Everitt, B. J. \& Robbins, T. W. (2011). Impulsivity, compulsivity, and top-down cognitive control. Neuron, 69, 680-694.

de Wit, H., Crean, J. \& Richards, J. B. (2000). Effects of d-amphetamine and ethanol on a measure of behavioral inhibition in humans. Behavioral Neuroscience, 114, 830-837.

de Wit, H. \& Richards, J. B. (2004). Dual determinants of drug use in humans: Reward and impulsivity. In R. A. Bevins \& M. T. Bardo (Eds.), Motivational factors in the etiology of drug abuse (Vol. 50, pp. 19-55).

Diamantopoulous, A. \& Siguaw, J. A. (2000). Introducing lisrel. London: Sage Publications.

Dickman, S. J. (1990). Functional and dysfunctional impulsivity - personality and cognitive correlates. Journal of Personality and Social Psychology, 58, 95-102.

Dom, G., D'Haene, P., Hulstijn, W. \& Sabbe, B. (2006). Impulsivity in abstinent early- and late-onset alcoholics: Differences in self-report measures and a discounting task. Addiction, 101, 50-59.

Donnellan, M. B., Conger, R. D. \& Burzette, R. G. (2007). Personality development from late adolescence to young adulthood: Differential stability, normative maturity, and evidence for the maturity-stability hypothesis. Journal of Personality, 75, 237-263. 
Dunn, O. J. \& Clark, V. (1969). Correlation coefficients measured on the same individuals. Journal of the American Statistical Association, 64, 366-377.

Eysenck, S. B. G. \& Eysenck, H. J. (1978). Impulsiveness and venturesomeness - their position in a dimensional system of personality description. Psychological Reports, 43, 12471255.

Field, M., Wiers, R. W., Christiansen, P., Fillmore, M. T. \& Verster, J. C. (2010). Acute alcohol effects on inhibitory control and implicit cognition: Implications for loss of control over drinking. Alcoholism-Clinical and Experimental Research, 34, 1346-1352.

Fillmore, M. T. \& Vogel-Sprott, M. (1999). An alcohol model of impaired inhibitory control and its treatment in humans. Experimental and Clinical Psychopharmacology, 7, 4955.

Fraley, R. C. \& Roberts, B. W. (2005). Patterns of continuity: A dynamic model for conceptualizing the stability of individual differences in psychological constructs across the life course. Psychological Review, 112, 60-74.

George, D. \& Mallery, P. (2003). Spss for windows step by step: A simple guide and reference. 11.0 update. (4th ed.). Boston: Allyn \& Bacon.

Gray, J. A. \& McNaughton, N. (2000). The neuropsychology of anxiety: An enquiry into the functions of the septo-hippocampal system (2nd ed.). Oxford: Oxford University Press.

Green, L., Myerson, J. \& Ostaszewski, P. (1999). Amount of reward has opposite effects on the discounting of delayed and probabilistic outcomes. Journal of Experimental Psychology-Learning Memory and Cognition, 25, 418-427.

Gullo, M. J. \& Dawe, S. (2008). Impulsivity and adolescent substance use: Rashly dismissed as "all-bad"? Neuroscience and Biobehavioral Reviews, 32, 1507-1518.

Hair, J. F. J., Black, W. C., Babin, B., J. \& Anderson, R. E. (2008). Multivariate data analysis: A global perspective (7th ed.). United States of America: Pearson Education.

Holden, R. R., Fekken, G. C. \& Cotton, D. H. (1991). Assessing psychopathology using structured test-item response latencies. Psychological Assessment: A Journal of Consulting and Clinical Psychology, 3, 111.

Horn, J. L. (1965). A rationale and test for the number of factors in factor-analysis. Psychometrika, 30, 179-185. 
Hu, L.-t. \& Bentler, P. M. (1999). Cutoff criteria for fit indexes in covariance structure analysis: Conventional criteria versus new alternatives. Structural Equation Modeling-a Multidisciplinary Journal, 6, 1-55.

Kaiser, H. (1974). An index of factorial simplicity. Psychometrika, 39, 31-36.

Kolin, E. A., Price, L. \& Zoob, I. (1964). Development of a sensation-seeking scale. Journal of Consulting Psychology, 28, 477-482.

Littlefield, A. K., Sher, K. J. \& Steinley, D. (2010). Developmental trajectories of impulsivity and their association with alcohol use and related outcomes during emerging and young adulthood i. Alcoholism-Clinical and Experimental Research, 34, 1409-1416.

Littlefield, A. K., Sher, K. J. \& Wood, P. K. (2009). Is "maturing out" of problematic alcohol involvement related to personality change? Journal of Abnormal Psychology, 118, 360-374.

Loeber, S. \& Duka, T. (2009). Acute alcohol decreases performance of an instrumental response to avoid aversive consequences in social drinkers. Psychopharmacology, 205, 577-587.

Logan, G. D., Schachar, R. J. \& Tannock, R. (1997). Impulsivity and inhibitory control. Psychological Science, 8, 60-64.

MacCallum, R. C., Browne, M. W. \& Sugawara, H. M. (1996). Power analysis and determination of sample size for covariance structure modeling. Psychological Methods, 1, 130-149.

Marczinski, C. A., Abroms, B. D., Van Selst, M. \& Fillmore, M. T. (2005). Alcohol-induced impairment of behavioral control: Differential effects on engaging vs. Disengaging responses. Psychopharmacology, 182, 452-459.

McCrae, R. R., Costa, P. T., Ostendorf, F., Angleitner, A., Hrebickova, M., Avia, M. D., Sanz, J., Sanchez-Bernardos, M. L., Kusdil, M. E., Woodfield, R., Saunders, P. R. \& Smith, P. B. (2000). Nature over nurture: Temperament, personality, and life span development. Journal of Personality and Social Psychology, 78, 173-186.

Miller, M. A. \& Fillmore, M. T. (2013). Protracted impairment of impulse control under an acute dose of alcohol: A time-course analysis. Addict Behav.

Newman, J. P., Widom, C. S. \& Nathan, S. (1985). Passive-avoidance in syndromes of disinhibition - psychopathy and extraversion. Journal of Personality and Social Psychology, 48, 1316-1327. 
Nunnally, J. \& Bernstein, I. (1994). Psychometric theory, 3rd ed.,. In: New York, NY: McGrawHill.

O'Connor, B. P. (2000). Spss and sas programs for determining the number of components using parallel analysis and velicer's map test. Behavior Research Methods Instruments \& Computers, 32, 396-402.

Patton, J. H., Stanford, M. S. \& Barratt, E. S. (1995). Factor structure of the barratt impulsiveness scale. J Clin Psychol, 51, 768-774.

Poltavski, D. V. \& Weatherly, J. N. (2013). Delay and probability discounting of multiple commodities in smokers and never-smokers using multiple-choice tasks. Behavioural pharmacology, 24, 659-667.

Quinn, P. D., Stappenbeck, C. A. \& Fromme, K. (2011). Collegiate heavy drinking prospectively predicts change in sensation seeking and impulsivity. Journal of Abnormal Psychology, 120, 543-556.

Robbins, T. W., Curran, H. V. \& de Wit, H. (2012). Special issue on impulsivity and compulsivity. Psychopharmacology, 219, 251-252.

Roberts, B. W., Caspi, A. \& Moffitt, T. E. (2001). The kids are alright: Growth and stability in personality development from adolescence to adulthood. Journal of Personality and Social Psychology, 81, 670-683.

Roberts, B. W., Caspi, A. \& Moffitt, T. E. (2003). Work experiences and personality development in young adulthood. Journal of Personality and Social Psychology, 84, 582-593.

Roberts, B. W. \& DelVecchio, W. F. (2000). The rank-order consistency of personality traits from childhood to old age: A quantitative review of longitudinal studies. Psychological Bulletin, 126, 3-25.

Rose, A. K. \& Duka, T. (2008). Effects of alcohol on inhibitory processes. Behavioural pharmacology, 19, 284-291.

Smith, C. L. \& Hantula, D. A. (2008). Methodological considerations in the study of delay discounting in intertemporal choice: A comparison of tasks and modes. Behavior Research Methods, 40, 940-953.

Spielberger, C. D. (1983). Manual for the state-trait anxiety inventory (form y). Palo Alto, CA: Mind Garden. 
Steiger, J. H. (1980). Tests for comparing elements of a correlation matrix. Psychological Bulletin, 87, 245-251.

Tabachnick, B. G. \& Fidell, L. S. (2007). Using multivariate statistics (5th ed.). Boston, MA: Pearson Education.

Torrubia, R., Avila, C., Molto, J. \& Caseras, X. (2001). The sensitivity to punishment and sensitivity to reward questionnaire (spsrq) as a measure of gray's anxiety and impulsivity dimensions. Personality and Individual Differences, 31, 837-862.

Vaidya, J. G., Gray, E. K., Haig, J. R., Mroczek, D. K. \& Watson, D. (2008). Differential stability and individual growth trajectories of big five and affective traits during young adulthood. Journal of Personality, 76, 267-304.

Velicer, W. (1976). Determining the number of components from the matrix of partial correlations. Psychometrika, 41, 321-327.

Weafer, J. \& de Wit, H. (2013). Sex differences in impulsive action and impulsive choice. Addict Behav.

Whiteside, S. P. \& Lynam, D. R. (2001). The five factor model and impulsivity: Using a structural model of personality to understand impulsivity. Personality and Individual Differences, 30, 669-689. 